\title{
An LMI approach for observer design for Takagi- Sugeno descriptor models
}

\author{
Víctor Estrada-Manzo, Thierry Marie Guerra \\ LAMIH UMR CNRS 8201 \\ University of Valenciennes and Hainaut-Cambrésis \\ Le Mont Houy, 59300 \\ Valenciennes, France \\ \{victor.estradamanzo, guerra\}@univ-valenciennes.fr
}

\author{
Zsófia Lendek \\ Department of Automation Technical University of Cluj- \\ Napoca \\ Memorandumului 28, 400114, \\ Cluj-Napoca, Romania \\ zsofia.lendek@aut.utcluj.ro
}

\begin{abstract}
This work presents a novel observer design for nonlinear descriptor systems using their Takagi-Sugeno representation. The approach allows obtaining more relaxed results than previous literature by changing the extended estimated state vector; this step permits using a full observer gain. The obtained conditions are pure LMI. Moreover, previous results are always included by the new ones. An example illustrates the benefits of the proposed method.
\end{abstract}

Keywords-Linear matrix inequality; nonlinear descriptor; Takagi-Sugeno models; observer design.

\section{INTRODUCTION}

A large class of nonlinear systems can be represented as Takagi-Sugeno (TS) models [1]. A TS model can exactly represent a nonlinear one via a systematic procedure called sector nonlinearity approach [2]. Once the TS model is obtained, it is seen as a collection of linear local models blended together by nonlinear membership functions (MFs) [3]. This structure allows using the direct Lyapunov method in order to perform stability analysis, controller/observer design; where the goal is to obtain conditions in terms of linear matrix inequalities (LMIs) [4]. Nonetheless, the TS-LMI framework has shortcomings. One of them is that the number of rules is exponential in the number of nonlinear terms, thus the number of LMI constrains grows exponentially. This issue can lead to an intractable problem.

In [5] the TS model was introduced to describe a more general one: the nonlinear descriptor model [6], [7]. Using the TS descriptor model the number of LMI conditions is smaller than using the standard TS [8]-[10]. Other authors have taken advantage of the so-called descriptor redundancy [11], [12].

When using state feedback techniques it is necessary to have all the states available; generally, this condition is unattainable; thus an observer must be employed [13]-[15]. For standard TS models there exist some results [15] while for TS descriptor ones a few works exist [9], [16]. These express the observer design conditions in terms of bilinear matrix inequalities (BMIs).

The aim of the present work is to improve existing results by obtaining pure LMI formulation, thus making the result less conservative. Moreover, a new observer scheme is possible with a full observer gain. These improvements are possible thanks to a new extended estimated state vector.

The paper is divided as follows: Section 2 defines the TS descriptor model, gives some useful notations and illustrates the motivation for this study; Section 3 states previous developments on the subject; Section 4 presents the main results on the observer design for TS descriptor models and shows the advantages via a numerical example; Section 5 concludes the paper.

\section{TS DESCRIPTOR MODEL AND NOTATION} [6]:

Consider the following nonlinear model in descriptor form

$$
\begin{aligned}
E(x) \dot{x}(t) & =A(x) x(t)+B(x) u(t) \\
y(t) & =C(x) x(t),
\end{aligned}
$$

where $x(t) \in \mathbb{R}^{n}$ is the state vector, $u(t) \in \mathbb{R}^{m}$ the control input, and $y(t) \in \mathbb{R}^{o}$ the output vector. Matrices $A(x)$, $B(x), C(x)$, and $E(x)$ are assumed to be smooth in a compact set $\Omega$ of the state space; matrix $E(x)$ is assumed to be nonsingular. This is motivated by mechanical models where the matrix $E(x)$ includes the inertia matrix, thus (1) is an ODE [9], [17].

Via the sector nonlinearity approach [3], the following TS descriptor model is an exact representation of (1) in the compact set $\Omega[8]$ :

$$
\begin{aligned}
\sum_{k=1}^{r_{e}} v_{k}(z(t)) E_{k} \dot{x}(t) & =\sum_{i=1}^{r} h_{i}(z(t))\left(A_{i} x(t)+B_{i} u(t)\right) \\
y(t) & =\sum_{i=1}^{r} h_{i}(z(t)) C_{i} x(t),
\end{aligned}
$$

where $\left(A_{i}, B_{i}, C_{i}\right)$ and $E_{k}$ represent the $i$-th linear right-hand side model and the $k$-th linear left-hand side model of the TS descriptor model, respectively. The $p$ nonlinear terms in the right-hand side of (1) are captured via the membership functions (MFs) $h_{i}(z(t)) \geq 0, i \in\{1, \ldots, r\}, r=2^{p}$; similar,

This work is supported by the Ministry of Higher Education and Research, the National Center for Scientific Research, the Nord-Pas-de-Calais Region, and a grant of the Romanian National Authority for Scientific Research, CNCS- UEFISCDI, project number PN-II-RU-TE-2011-3-0043, contract number 74/05.10.2011. The authors gratefully acknowledge the support of these institutions.

978-1-4799-3732-5/14/\$31.00 @2014 IEEE 
the $p_{e}$ nonlinear terms on the left-hand side of (1) give $v_{k}(z(t)) \geq 0, k \in\left\{1, \ldots, r_{e}\right\}, r_{e}=2^{p_{e}}$. These MFs hold the convex-sum property $\quad \sum_{i=1}^{r} h_{i}(z(t))=1, \quad h_{i}(z(t)) \geq 0$, $\sum_{k=1}^{r_{e}} v_{k}(z(t))=1, v_{k}(z(t)) \geq 0$ in a compact set of the state space $\Omega$ [3]. In this work the premise vector $z(t)$ only depends on measured variables.

The following example motives us to keep the nonlinear descriptor form instead of calculating the standard state space form $\dot{x}=A(x) x+B(x) u$.

Example 1. Consider a system in nonlinear natural form (1) with $A(x)=\left[\begin{array}{cc}0.1 & 0.9 \\ \cos \left(x_{2}\right) & -1.4\end{array}\right], C(x)=\left[\begin{array}{c}0 \\ \sin \left(x_{2}\right) / x_{2}+1\end{array}\right]$, $B(x)=\left[\begin{array}{l}0 \\ 1\end{array}\right]$, and $E(x)=\left[\begin{array}{cc}1 & -x_{2}^{2} \\ x_{2}^{2} & 1\end{array}\right]$. The representation in the form (2) gives $r_{e}=2$ and $r=4$ due to the number of nonlinearities in the left-hand side and right-hand side. To rewrite (2) into the standard TS representation it is necessary to invert the matrix $E(x)$, resulting in $\dot{x}=(E(x))^{-1} A(x)+(E(x))^{-1} B(x) u$, with

$$
(E(x))^{-1}=\left[\begin{array}{cc}
\frac{1}{1+x_{2}^{4}} & \frac{x_{2}^{2}}{1+x_{2}^{4}} \\
-\frac{x_{2}^{2}}{1+x_{2}^{4}} & \frac{1}{1+x_{2}^{4}}
\end{array}\right] .
$$

Therefore, four different nonlinearities have to be considered, resulting in $r=2^{4}$ due to all the nonlinearities on the right-hand side. Under the quadratic framework [3], [16], the number of LMI conditions to be verified for the standard TS model is 257 while for the TS descriptor one is $33 . \diamond$

The following shorthand notations will be employed:

$$
\begin{gathered}
\Upsilon_{h}=\sum_{i=1}^{r} h_{i}(z(t)) \Upsilon_{i}, \quad \Upsilon_{h}^{-1}=\left(\sum_{i=1}^{r} h_{i}(z(t)) \Upsilon_{i}\right)^{-1}, \\
\Upsilon_{h h v}=\sum_{i=1}^{r} \sum_{j=1}^{r} h_{i}(z(t)) h_{j}(z(t)) v_{k}(z(t)) \Upsilon_{i j}^{k} .
\end{gathered}
$$

Indices may change to $v$ if the respective MF is $v_{k}$. An asterisk $(*)$ will be used in matrix expressions to denote the transpose of the symmetric element; for in-line expressions it will denote the transpose of the terms on its left side. Arguments will be omitted when their meaning is clear.

Employing the above definitions the TS descriptor model (2) writes:

$$
\begin{aligned}
E_{v} \dot{x} & =A_{h} x+B_{h} u \\
y & =C_{h} x .
\end{aligned}
$$

While designing conditions for TS descriptor models, the following extended state vector is commonly used $\bar{x}=\left[\begin{array}{ll}x^{T} & \dot{x}^{T}\end{array}\right]^{T}$. Then (3) can be written as:

$$
\begin{aligned}
\bar{E} \dot{\bar{x}} & =\bar{A}_{h v} \bar{x}+\bar{B}_{h} u \\
y & =\bar{C}_{h} \bar{x},
\end{aligned}
$$

with $\quad \bar{E}=\left[\begin{array}{ll}I & 0 \\ 0 & 0\end{array}\right], \quad \bar{A}_{h v}=\left[\begin{array}{cc}0 & I \\ A_{h} & -E_{v}\end{array}\right], \quad \bar{B}_{h}=\left[\begin{array}{c}0 \\ B_{h}\end{array}\right], \quad$ and $\bar{C}_{h}=\left[\begin{array}{ll}C_{h} & 0\end{array}\right]$.

The following Lemma provides a way to drop off the MFs in order to obtain an LMI formulation.

Relaxation Lemma [18]: Let $\Upsilon_{i j}^{k}$ be matrices of appropriate dimensions. Then

$$
\sum_{i=1}^{r} \sum_{j=1}^{r} \sum_{k=1}^{r_{e}} h_{i} h_{j} v_{k} \Upsilon_{i j}^{k}<0
$$

holds if

$$
\begin{aligned}
& \Upsilon_{i i}^{k}<0, \quad \forall i, \\
& \frac{2}{r-1} \Upsilon_{i i}^{k}+\Upsilon_{i j}^{k}+\Upsilon_{j i}^{k}<0, \quad i \neq j,
\end{aligned}
$$

for $i, j \in\{1, \ldots, r\}, k \in\left\{1, \ldots, r_{e}\right\}$.

The next section presents previous results in the literature.

\section{PREVIOUS RESULTS}

Previously, in [9], [16] the following observer for the model (4) was proposed.

$$
\begin{aligned}
\bar{E}_{\dot{\hat{x}}}^{*} & =\bar{A}_{h v} \hat{\bar{x}}^{*}+\bar{B}_{h} u+\bar{L}_{h v}(y-\hat{y}) \\
\hat{y} & =\bar{C}_{h} \hat{\bar{x}}^{*},
\end{aligned}
$$

with $\hat{\hat{x}}^{*}=\left[\begin{array}{cc}\hat{x}^{T} & \dot{\hat{x}}^{T}\end{array}\right]^{T}$ being the extended estimated state vector and $\bar{L}_{h v}=\left[\begin{array}{ll}0 & L_{h v}^{T}\end{array}\right]^{T}$ being the observer gain.

The main task is to make the estimation error $e=x-\hat{x}$ tend to zero when $t \rightarrow \infty$. For this purpose an extended estimation error is defined: $e^{*}=\bar{x}-\hat{\bar{x}}^{*}=\left[\begin{array}{c}x-\hat{x} \\ \dot{x}-\dot{\hat{x}}\end{array}\right]$ and its dynamic is given as

$$
\begin{aligned}
\bar{E} \dot{e}^{*} & =\bar{A}_{h v} \bar{x}+\bar{B}_{h} u-\left(\bar{A}_{h v} \hat{\bar{x}}^{*}+\bar{B}_{h} u+\bar{L}_{h v}(y-\hat{y})\right) \\
& =\bar{A}_{h v}\left(\bar{x}-\hat{\bar{x}}^{*}\right)-\bar{L}_{h v} \bar{C}_{h}\left(\bar{x}-\hat{\bar{x}}^{*}\right) .
\end{aligned}
$$

Finally,

$$
\bar{E} \dot{e}^{*}=\left(\bar{A}_{h v}-\bar{L}_{h v} \bar{C}_{h}\right) e^{*} .
$$

Then, employing a quadratic Lyapunov function candidate 


$$
V\left(e^{*}\right)=e^{* T} \bar{E} P e^{*} ; \quad \bar{E} P=P^{T} \bar{E}^{T},
$$

with $P=\left[\begin{array}{ll}P_{1} & 0 \\ P_{3} & P_{4}\end{array}\right]$, this procedure leads to the following result:

Lemma 1 [9], [16]. Consider the system (4) together with the observer (7). If there exist matrices $P_{1}=P_{1}^{T}>0, P_{3}$, and $M_{j k}, j \in\{1, \ldots, r\}, k \in\left\{1, \ldots, r_{e}\right\}$ such that (6) holds with

$$
\Upsilon_{i j}^{k}=\left[\begin{array}{cc}
P_{3}^{T} A_{i}-M_{j k} C_{i}+(*) & (*) \\
P_{3}^{T} A_{i}-M_{j k} C_{i}+P_{1}-E_{k}^{T} P_{3} & -E_{k}^{T} P_{3}-P_{3}^{T} E_{k}
\end{array}\right],
$$

for $i, j \in\{1, \ldots, r\}, k \in\left\{1, \ldots, r_{e}\right\}$, the estimation error $e$ is asymptotically stable.

Proof: The derivative of the Lyapunov function (10) writes

$$
\begin{aligned}
\dot{V}\left(e^{*}\right) & =e^{* T} \bar{E} P e^{*}+e^{* T} P^{T} \bar{E} \dot{e}^{*} \\
& =e^{* T} P^{T}\left(\bar{A}_{h v}-\bar{L}_{h v} \bar{C}_{h}\right) e^{*}+\left({ }^{*}\right) .
\end{aligned}
$$

To ensure that $\dot{V}\left(e^{*}\right)<0$ a sufficient conditions is

$$
P^{T} \bar{A}_{h v}-P^{T} \bar{L}_{h v} \bar{C}_{h}+(*)<0 .
$$

Developing (13) gives

$$
\left[\begin{array}{cc}
P_{3}^{T} A_{h}-P_{3}^{T} L_{h v} C_{h}+(*) & (*) \\
P_{4}^{T} A_{h}-P_{4}^{T} L_{h v} C_{h}+P_{1}-E_{v}^{T} P_{3} & -E_{v}^{T} P_{4}-P_{4}^{T} E_{v}
\end{array}\right]<0 .
$$

Note that (14) cannot be written as an LMI. Setting $P_{3}=P_{4}$ and defining $M_{h v}=P_{3}^{T} L_{h v}$ as in [16] yields

$$
\left[\begin{array}{cc}
P_{3}^{T} A_{h}-M_{h v} C_{h}+(*) & (*) \\
P_{3}^{T} A_{h}-M_{h v} C_{h}+P_{1}-E_{v}^{T} P_{3} & -E_{v}^{T} P_{3}-P_{3}^{T} E_{v}
\end{array}\right]<0 .
$$

Finally, applying the Relaxation Lemma the proof is concluded. $\square$

Remark 1: Using inequality (14) one cannot obtain an LMI condition because of the terms $P_{3}^{T} L_{h v} C_{h}$ and $P_{4}^{T} L_{h v} C_{h}$. In [16] one way is to chose $P_{3}=P_{4}$; in [9], the authors suggest the pole placement technique and once the gain $L_{h v}$ is designed, the expression (14) is used to verify convergence of the estimation error.

The next section shows a way to obtain a pure LMI solution to solve the observer problem by changing the extended estimated state vector.

\section{MAIN RESULTS}

This section presents an observer design via LMIs with a full observer gain $\bar{L}_{h v}=\left[\begin{array}{l}L_{1 h v} \\ L_{2 h v}\end{array}\right]$.
A new way to write an observer for the model (4) is using a new estimated state vector $\hat{\bar{x}}=\left[\begin{array}{l}\hat{x} \\ \alpha\end{array}\right]$. Then

$$
\begin{aligned}
\bar{E} \dot{\hat{x}} & =\bar{A}_{h v} \hat{\bar{x}}+\bar{B}_{h} u+P^{-T} \bar{L}_{h v}(y-\hat{y}) \\
\hat{y} & =\bar{C}_{h} \hat{\bar{x}},
\end{aligned}
$$

with the structure of $P=\left[\begin{array}{cc}P_{1} & 0 \\ P_{3} & P_{4}\end{array}\right], P_{1}=P_{1}^{T}>0, P_{4}$ being a regular matrix; moreover $P^{-1}=\left[\begin{array}{cc}P_{1}^{-1} & 0 \\ -P_{4}^{-1} P_{3} P_{1}^{-1} & P_{4}^{-1}\end{array}\right]$ and a new observer gain $\bar{L}_{h v}=\left[\begin{array}{c}L_{1 h v} \\ L_{2 h v}\end{array}\right]$ can be considered.

The small change in the estimated state vector $\hat{\bar{x}}$ allows rewriting the final form of the observer with a new structure of $\bar{L}_{h v}$.

An extended estimation error is defined: $\bar{e}=\bar{x}-\hat{\bar{x}}=\left[\begin{array}{l}x-\hat{x} \\ \dot{x}-\alpha\end{array}\right]$ and its dynamics as follows

$$
\begin{aligned}
\bar{E} \dot{\bar{e}} & =\bar{A}_{h v} \bar{x}+\bar{B}_{h} u-\left(\bar{A}_{h v} \hat{\bar{x}}+\bar{B}_{h} u+P^{-T} \bar{L}_{h v}(y-\hat{y})\right) \\
& =\bar{A}_{h v}(\bar{x}-\hat{\bar{x}})-P^{-T} \bar{L}_{h v} \bar{C}_{h}(\bar{x}-\hat{\bar{x}}) .
\end{aligned}
$$

Finally,

$$
\bar{E} \dot{\bar{e}}=\left(\bar{A}_{h v}-P^{-T} \bar{L}_{h v} \bar{C}_{h}\right) \bar{e} .
$$

Consider the following Lyapunov function candidate

$$
V(\bar{e})=\bar{e}^{T} \bar{E} P \bar{e} ; \quad \bar{E} P=P^{T} \bar{E}^{T},
$$

where the matrix $P$ has the same structure as in (10).

Then, the following result can be stated.

Theorem 1: Consider the system (4) together with the observer (16). If there exist matrices $P_{1}=P_{1}^{T}>0, P_{3}, P_{4}, L_{1 j k}$ and $L_{2 j k}, j \in\{1, \ldots, r\}, k \in\left\{1, \ldots, r_{e}\right\}$ such that (6) holds with

$$
\Upsilon_{i j}^{k}=\left[\begin{array}{cc}
P_{3}^{T} A_{i}+L_{1 j k} C_{i}+(*) & (*) \\
P_{4}^{T} A_{i}-L_{2 j k} C_{i}+P_{1}-E_{k}^{T} P_{3} & -E_{k}^{T} P_{4}-P_{4}^{T} E_{k}
\end{array}\right],
$$

for $i, j \in\{1, \ldots, r\}, k \in\left\{1, \ldots, r_{e}\right\}$.

Then, the estimation error $e$ is asymptotically stable. The observer structure is

$$
\begin{aligned}
E_{v} \dot{\hat{x}} & =A_{h} \hat{x}+B_{h} u+\left[\begin{array}{ll}
E_{v} & I
\end{array}\right] P^{-T}\left[\begin{array}{l}
L_{1 h v} \\
L_{2 h v}
\end{array}\right](y-\hat{y}) \\
\hat{y} & =C_{h} \hat{x} .
\end{aligned}
$$


Proof: The derivative of the Lyapunov function (19) writes

$$
\begin{aligned}
\dot{V}(\bar{e}) & =\dot{\bar{e}} \bar{E} P \bar{e}+\bar{e}^{T} P^{T} \bar{E} \dot{\bar{e}} \\
& =\bar{e}^{T} P^{T}\left(\bar{A}_{h v}-P^{-T} \bar{L}_{h v} \bar{C}_{h}\right) \bar{e}+\left({ }^{*}\right) .
\end{aligned}
$$

To ensure that $\dot{V}(\bar{e})<0$ a sufficient conditions is

$$
P^{T} \bar{A}_{h v}-\bar{L}_{h v} \bar{C}_{h}+(*)<0 .
$$

Expanding (23) gives

$$
\Upsilon_{h h}^{v}=\left[\begin{array}{cc}
P_{3}^{T} A_{h}-L_{1 h v} C_{h}+(*) & (*) \\
P_{4}^{T} A_{h}-L_{2 h v} C_{h}+P_{1}-E_{v}^{T} P_{3} & -P_{4}^{T} E_{v}-(*)
\end{array}\right]<0 .
$$

To obtain the final form (21), recall (16) which after some substitutions yields

$$
\begin{gathered}
{\left[\begin{array}{cc}
I & 0 \\
0 & 0
\end{array}\right]\left[\begin{array}{c}
\hat{\hat{x}} \\
\dot{\alpha}
\end{array}\right]=\left[\begin{array}{cc}
0 & I \\
A_{h} & -E_{v}
\end{array}\right]\left[\begin{array}{l}
\hat{x} \\
\alpha
\end{array}\right]+\left[\begin{array}{c}
0 \\
B_{h}
\end{array}\right] u} \\
+P^{-T}\left[\begin{array}{l}
L_{1 h v} \\
L_{2 h v}
\end{array}\right](y-\hat{y}) .
\end{gathered}
$$

After regrouping some terms, it yields

$$
\begin{aligned}
{\left[\begin{array}{cc}
I & 0 \\
0 & 0
\end{array}\right]\left[\begin{array}{c}
\dot{\hat{x}} \\
\dot{\alpha}
\end{array}\right]=} & \left(\left[\begin{array}{cc}
0 & I \\
A_{h} & -E_{v}
\end{array}\right]-P^{-T}\left[\begin{array}{l}
L_{1 h v} \\
L_{2 h v}
\end{array}\right]\left[\begin{array}{ll}
C_{h} & 0
\end{array}\right]\right)\left[\begin{array}{c}
\hat{x} \\
\alpha
\end{array}\right] \\
& +\left[\begin{array}{c}
0 \\
B_{h}
\end{array}\right] u+P^{-T}\left[\begin{array}{l}
L_{1 h v} \\
L_{2 h v}
\end{array}\right] y .
\end{aligned}
$$

Define the vector:

$$
\begin{aligned}
{\left[\begin{array}{c}
\mathcal{K}_{h h v} \\
\mathcal{K}_{2 h v}
\end{array}\right] } & =\left[\begin{array}{cc}
P_{1}^{-1} & -P_{1}^{-1} P_{3}^{T} P_{4}^{-T} \\
0 & P_{4}^{-T}
\end{array}\right]\left[\begin{array}{l}
L_{1 h v} \\
L_{2 h v}
\end{array}\right] \\
& =\left[\begin{array}{c}
P_{1}^{-1}\left(L_{1 h v}-P_{3}^{T} P_{4}^{-T} L_{2 h v}\right) \\
P_{4}^{-1} L_{2 h v}
\end{array}\right],
\end{aligned}
$$

thus (25) writes:

$$
\left[\begin{array}{cc}
I & 0 \\
0 & 0
\end{array}\right]\left[\begin{array}{c}
\dot{\hat{x}} \\
\dot{\alpha}
\end{array}\right]=\left[\begin{array}{cc}
-\mathcal{K}_{1 h} C_{h} & I \\
A_{h}-\mathcal{K}_{2 h v} C_{h} & -E_{v}
\end{array}\right]\left[\begin{array}{c}
\hat{x} \\
\alpha
\end{array}\right]+\left[\begin{array}{c}
0 \\
B_{h}
\end{array}\right] u+\left[\begin{array}{c}
\mathcal{K}_{1 h v} \\
\mathcal{K}_{2 h v}
\end{array}\right] y,
$$

or equivalently

$$
\begin{aligned}
\dot{\hat{x}} & =\mathcal{K}_{1 h v}\left(y-C_{h} \hat{x}\right)+\alpha \\
E_{v} \alpha & =\left(A_{h}-\mathcal{K}_{2 h v} C_{h}\right) \hat{x}+B_{h} u+\mathcal{K}_{2 h v} y,
\end{aligned}
$$

and taking $\alpha=\dot{\hat{x}}-\mathcal{K}_{1 h v}\left(y-C_{h} \hat{x}\right)$, (28) gives

$$
\begin{aligned}
E_{v} \dot{\hat{x}}=\left(A_{h}\right. & \left.-\mathcal{K}_{2 h v} C_{h}\right) \hat{x}+B_{h} u \\
& +\mathcal{K}_{2 h v} y+E_{v} \mathcal{K}_{1 h v}\left(y-C_{h} \hat{x}\right) .
\end{aligned}
$$

or

$$
E_{v} \dot{\hat{x}}=A_{h} \hat{x}+B_{h} u+E_{v} \mathcal{K}_{h v}(y-\hat{y})+\mathcal{K}_{2 h v}(y-\hat{y}) .
$$

Regrouping the terms in (29) yields

$$
E_{v} \dot{\hat{x}}=A_{h} \hat{x}+B_{h} u+\left[\begin{array}{ll}
E_{v} & I
\end{array}\right]\left[\begin{array}{l}
\mathcal{K}_{1 h v} \\
\mathcal{K}_{2 h v}
\end{array}\right](y-\hat{y}) .
$$

Using the definition (27), (30) corresponds to (21), thus concluding the proof. $\square$

Remark 2: Note that without the new auxiliary variable $\alpha$, expression (28) does not hold unless a perfect observation is obtained. Moreover if the LMI problem is feasible, it means that $x-\hat{x} \rightarrow 0$ and $\dot{x}-\alpha \rightarrow 0$ as the time goes to infinity.

Corollary 1: Results in Theorem 1 always includes results in Lemma 1.

Proof: In (24), set $L_{1 h v}=L_{2 h v}=M_{h v}$ and $P_{3}=P_{4}$, thus resulting (15). $\square$

The following numerical example compares the performance of Theorem 1 and Lemma 1.

Example 2. Consider (2) with $u(t)=0$ with $r=r_{e}=2$ and its linear local matrices as follows:

$$
\begin{array}{cc}
A_{1}=\left[\begin{array}{cc}
-0.35 & 1.24+b \\
-0.74 & 0.5
\end{array}\right], & A_{2}=\left[\begin{array}{cc}
-0.35 & 1.24-b \\
-0.74 & 0.5
\end{array}\right], \\
E_{1}=\left[\begin{array}{cc}
0.37 & 0.43-a \\
0.23 & 1.15
\end{array}\right], \quad E_{2}=\left[\begin{array}{cc}
0.37 & 0.43+a \\
0.23 & 1.15
\end{array}\right], \\
C_{1}=\left[\begin{array}{c}
1.5+a \\
0
\end{array}\right], \quad C_{2}=\left[\begin{array}{c}
1.5-a \\
0
\end{array}\right]^{T} .
\end{array}
$$

With parameters $a \in[-1.5,1.5]$ and $b \in[-1.5,1.5]$.

The values of $a$ and $b$ for which observers can be designed (the LMI conditions are feasible) are illustrated in Figure 1.

Note that when $a=-1.2$ and $b=-0.3$ there is no solution for the conditions in Lemma 1 while Theorem 1 provides a solution. The following matrices were obtained:

$$
\begin{aligned}
& P_{1}=\left[\begin{array}{cc}
0.14 & -0.12 \\
-0.12 & 0.21
\end{array}\right], P_{3}=\left[\begin{array}{cc}
0.36 & -1 \\
0.92 & 0.93
\end{array}\right], P_{4}=\left[\begin{array}{cc}
0.31 & -0.16 \\
-0.16 & 1.60
\end{array}\right] \\
& L_{11}=\left[\begin{array}{c}
1.80 \\
-0.04 \\
-0.35 \\
-4.89
\end{array}\right], L_{12}=\left[\begin{array}{c}
-0.04 \\
0.86 \\
-0.29 \\
-2.78
\end{array}\right], L_{21}=\left[\begin{array}{c}
0.21 \\
0.36 \\
-0.06 \\
-0.80
\end{array}\right], L_{22}=\left[\begin{array}{c}
0.25 \\
0.17 \\
-0.05 \\
-0.57
\end{array}\right] .
\end{aligned}
$$

For simulation purposes the MFs are defined as follows: $v_{1}=\frac{1}{1+x_{1}^{2}}, \quad v_{2}=1-v_{1}, \quad h_{1}=\frac{\cos \left(x_{1}\right)+1}{2}, \quad$ and $\quad h_{2}=1-h_{1}$. Simulation results with initial conditions $x(0)=\left[\begin{array}{ll}-0.7 & 0.5\end{array}\right]^{T}$ and $\hat{x}(0)=\left[\begin{array}{ll}0 & 0\end{array}\right]^{T}$ are presented in Figures 2 and 3. 


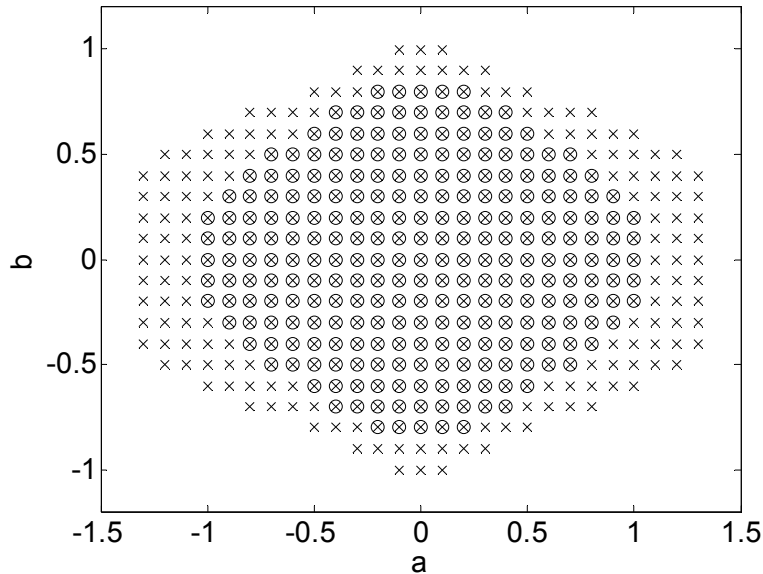

Fig. 1. Solution set for Lemma $1(O)$ and Theorem $1(\times)$.
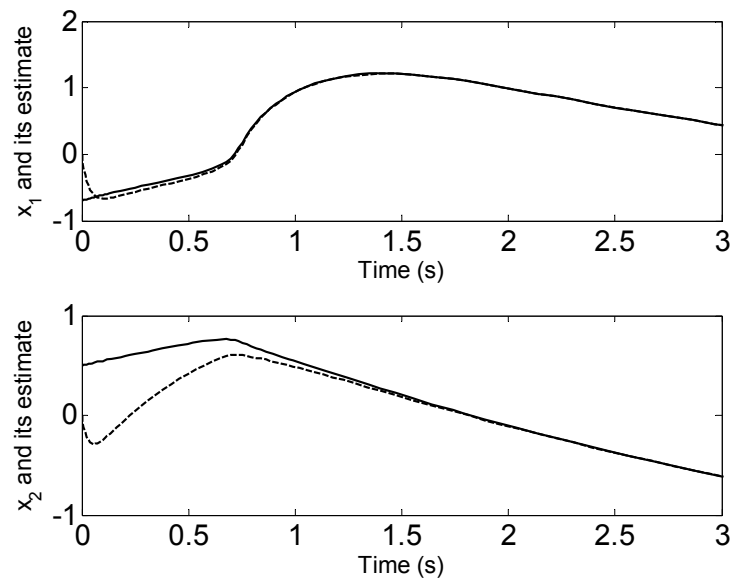

Fig. 2. States (solid lines) and their estimates (dashed lines) in Example 2.

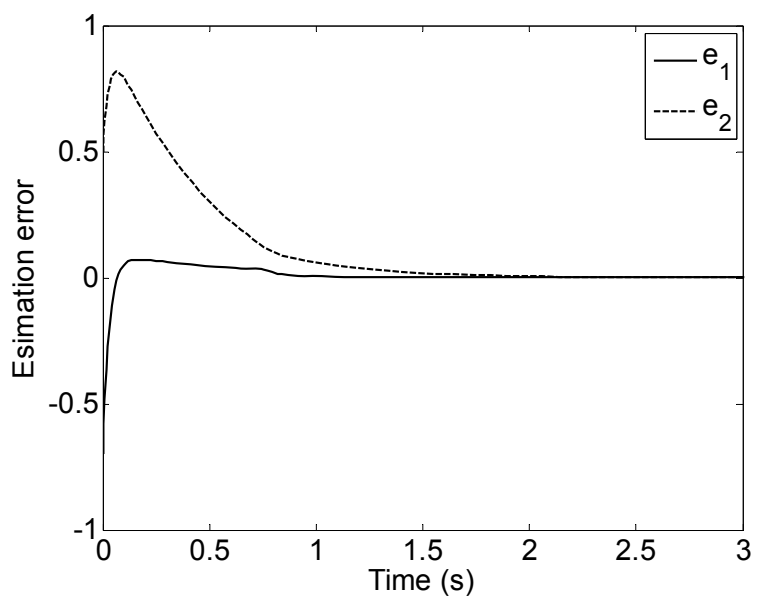

Fig. 3. Estimation error in Example 2.

\section{CONCLUSIONS}

In this paper, a new observer scheme for TS descriptor models has been presented. This scheme is based on a quadratic Lyapunov function together with a new extended estimated state vector, which allows using a full observer gain and obtaining LMI formulation. The effectiveness of the new approach is illustrated via an example.

\section{REFERENCES}

[1] T. Takagi and M. Sugeno, "Fuzzy identification of systems and its applications to modeling and control," IEEE Transactions on Systems, Man and Cybernetics, vol. 15, no. 1, pp. 116-132, 1985.

[2] H. Ohtake, K. Tanaka, and H. Wang, "Fuzzy modeling via sector nonlinearity concept," in Joint 9th IFSA World Congress and 20th NAFIPS International Conference, Vancouver, Canada, 2001, pp. $127-132$.

[3] K. Tanaka and H. O. Wang, Fuzzy Control Systems Design and Analysis: a Linear Matrix Inequality Approach. New York: John Wiley \& Sons, Inc., 2001.

[4] S. Boyd, L. El Ghaoul, E. Feron, and V. Balakrishnan, Linear matrix inequalities in system and control theory. Philadelphia, PA: Society for Industrial and Applied Mathematics, 1994.

[5] T. Taniguchi, K. Tanaka, K. Yamafuji, and H. O. Wang, "Fuzzy descriptor systems: stability analysis and design via LMIs," in Proceedings of the American Control Conference, California, USA, 1999, vol. 3, pp. 1827-1831.

[6] D. Luenberger, "Dynamic equations in descriptor form," IEEE Transactions on Automatic Control, vol. 22, no. 3, pp. 312-321, 1977.

[7] L. Dai, Singular control systems, vol. 118. Springer-Verlag, 1989.

[8] T. Taniguchi, K. Tanaka, and H. O. Wang, "Fuzzy descriptor systems and nonlinear model following control," IEEE Transactions on Fuzzy Systems, vol. 8, no. 4, pp. 442-452, 2000.

[9] K. Guelton, S. Delprat, and T. M. Guerra, "An alternative to inverse dynamics joint torques estimation in human stance based on a TakagiSugeno unknown-inputs observer in the descriptor form," Control Engineering Practice, vol. 16, no. 12, pp. 1414-1426, 2008.

[10] V. Estrada-Manzo, T. M. Guerra, Zs. Lendek, and M. Bernal, "Improvements on non-quadratic stabilization of continuous-time Takagi-Sugeno descriptor models," in 2013 IEEE International Conference on Fuzzy Systems, Hyderabad, India, 2013, pp. 1-6.

[11] K. Guelton, T. Bouarar, and N. Manamanni, "Robust dynamic output feedback fuzzy Lyapunov stabilization of Takagi-Sugeno systems-A descriptor redundancy approach," Fuzzy Sets and Systems, vol. 160, no. 19, pp. 2796-2811, 2009.

[12] M. Chadli and T. M. Guerra, "LMI solution for robust static output feedback control of Takagi-Sugeno fuzzy models," IEEE Transactions on Fuzzy Systems, vol. 20, no. 6, pp. 1160-1165, 2012.

[13] D. Luenberger, "Observers for multivariable systems," IEEE Transactions on Automatic Control, vol. 11, no. 2, pp. 190-197, Apr. 1966.

[14] P. Bergsten and D. Driankov, "Observers for Takagi-Sugeno fuzzy systems," IEEE Transactions on Systems, Man and Cybernetics, Part $B$, vol. 32, no. 1, pp. 114-121, 2002.

[15] Zs. Lendek, T. M. Guerra, R. Babuška, and B. De Schutter, Stability analysis and nonlinear observer design using Takagi-Sugeno fuzzy models, vol. 262. Germany: Springer, 2010.

[16] T. M. Guerra, K. Guelton, and S. Delprat, "A class of nonlinear observers in descriptor form: LMI based design with applications in biomechanics," in 2nd IFAC Workshop on Advanced Fuzzy/Neural Control, Oulu, Finland, 2004, vol. 4.

[17] L. Vermeiren, A. Dequidt, M. Afroun, and T. M. Guerra, "Motion control of planar parallel robot using the fuzzy descriptor system approach," ISA Transactions, vol. 51, pp. 596-608, 2012.

[18] H. D. Tuan, P. Apkarian, T. Narikiyo, and Y. Yamamoto, "Parameterized linear matrix inequality techniques in fuzzy control system design," IEEE Transactions on Fuzzy Systems, vol. 9, no. 2, pp. 324-332, 2001. 